\title{
Review of: "Successful Cessation Programs that Reduce Comorbidity may Explain Surprisingly Low Smoking Rates among Hospitalized COVID-19 Patients"
}

Joe G. Gitchell ${ }^{1}$

1 Pinney Associates

Potential competing interests: My employer, PinneyAssociates, provides consulting services regarding tobacco harm minimization and vaping products to JUUL Labs, Inc, on an exclusive basis. I also own an interest in a nicotine gum that has not been developed nor commercialized.

I read this paper with interest. While I have not been attending to every preprint and publication in the area of smoking/nicotine and coronavirus, I have seen and read many. This is the first time that I have read the "smoking cessation protection" hypothesis presented.

As I understand it in lay terms (and I hope the authors will correct my misunderstandings!), the posited mechanism by which smokers would be underrepresented in samples of COVID19 hospitalized patients is because smoking causes a number of diseases (eg, chronic lung and heart disease) that are 'primary' risk factors for COVID infection and progression, but that upon suffering these conditions, smokers are more likely to quit smoking (including as prompted by public health campaigns focused on raising awareness of health consequences of smoking). As a result, former smokers still experiencing conditions caused by smoking that put them at greater risk for infection and progression "enrich" the sample of nonsmoking patients presenting at health care settings.

The authors then recommend more intensive research to understand smoking and quitting histories among COVID19 patients to evaluate this "smoking cessation protection" hypothesis.

One analytic approach that could be pursued with existing data (and while I had thought I had seen a publication in this vein, the closest thing I could find with a quick search was a discussion of smokers living with morbidities in the 2014 Surgeon General's Report (Chapter 12, section starting on page 667)) is to examine whether smokers living with smoking-related conditions are more likely to stop smoking than those without them? NHANES would seem to be a useful dataset, but PATH could also be informative. These analyses will not really allow for understanding causality or mechanisms but could contribute further contextual evidence for this question.

I hope that increasing amounts of data are brought to bear on these questions.

\section{Disclosures}

My employer, PinneyAssociates, provides consulting services regarding tobacco harm minimization and vaping products to JUUL Labs, Inc, on an exclusive basis. I also own an interest in a nicotine gum that has not been developed nor 
commercialized. 